$09 ; 15$

\title{
Оптический квантовый термометр с субмикронным разрешением, основанный на явлении кросс-релаксации спиновых уровней
}

\author{
(C) А.Н. Анисимов, Р.А. Бабунц, М.В. Музафрарова, А.П. Бундакова, \\ В.А. Солтамов, П.Г. Баранов ฯ
}

Физико-технический институт им. А.Ф. Иоффе РАН, Санкт-Петербург, Россия

『 E-mail: pavel.baranov@mail.ioffe.ru

Поступило в Редакцию 21 мая 2018 г.

Предложен оптический квантовый термометр с субмикронным пространственным разрешением, основанный на физическом явлении оптического отклика в системе спиновых центров в карбиде кремния в условиях кросс-релаксации между оптически активными центрами в квадруплетном спиновом состоянии и триплетными центрами, у которых наблюдается аномально сильная зависимость расщепления тонкой структуры от температуры.

DOI: 10.21883/PJTF.2018.17.46568.17399

Измерение слабых температурных полей с высоким пространственным разрешением на уровне микро- и нанометров является важнейшей проблемой в различных областях (от фундаментальной физики и материаловедения до хранения данных и биомедицинской науки).

Были разработаны оптические квантовые термометры для измерения температур с субмикронным разрешением на основе азотновакансионных $(\mathrm{N} V)$ центров в алмазе, имеющих основное триплетное спиновое состояние, спиновые уровни которого селективно заселяются под действием оптического возбуждения [1]. Недавно нами были обнаружены вакансионные спиновые центры в карбиде кремния $(\mathrm{SiC})$, в которых спиновые уровни с $S=3 / 2$ селективно заселяются под действием оптического излучения в ближнем ИК-диапазоне, совместимом с полосой прозрачности волоконной оптики и биологических систем, и предложен способ использования оптически детектируемого магнит- 
ного резонанса (ОДМР) для измерения магнитных и температурных полей [2-9]. Был предложен оптический квантовый термометр [8], который использовал физическое явление сильного изменения интенсивности фотолюминесценции (ФЛ) (photoluminescence, PL) в магнитных полях, близких к точке антипересечения спиновых подуровней центров c $S=3 / 2$ (level anticrossing - LAC) в возбужденном состоянии, которые характеризовались сильной зависимостью расщепления тонкой структуры (zero-field splitting) от температуры [7]. Была достигнута точность измерения температуры до $50 \mathrm{mK} / \mathrm{Hz}^{1 / 2}$ с пространственным разрешением $\sim 300 \mathrm{~nm}$, соответствующим размеру пятна сфокусированного лазера конфокального микроскопа. Недостатком способа измерения температуры была сравнительно большая ширина линии ОДМР, достигающая $2 \mathrm{mT}$, которая определяется обратным временем жизни возбужденного состояния $\sim 6 \mathrm{~ns}$ и, следовательно, не могла быть уменьшена.

Задачей настоящей работы является разработка такого оптического квантового термометра, который бы позволил увеличить чувствительность. В $\mathrm{SiC}$ имеются дефекты со спином $S=1$, характеризующиеся чрезвычайно сильной зависимостью расщепления тонкой структуры [10], однако эти центры являются оптически неактивными (назовем

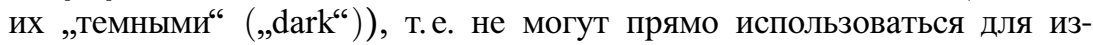
мерения температуры. Однако из-за диполь-дипольного взаимодействия с соседними оптически активными спиновыми центрами со спином $S=3 / 2$, (назовем их „яркими“ („bright“)) в магнитных полях, соответствующих совпадению разностей энергий спиновых уровней „темных“ и „ярких“ центров, наблюдается сильное изменение интенсивности ФЛ „,рких“ центров - так называемое явление кросс-релаксации (KP) (cross-relaxation, CR). Нами обнаружено, что ширина линии КР примерно на порядок меньше ширины линии антипересечения в возбужденном состоянии при одинаково сильной температурной зависимости положений обеих линий.

Спиновый гамильтониан для нахождения энергий спиновых уровней в магнитном поле имеет вид

$$
H=\gamma \mathbf{B} \cdot \mathbf{S}+D\left[S_{z}^{2}-(1 / 3) S(S+1)\right] .
$$

Здесь $\mathbf{B}-$ внешнее магнитное поле, $\mathbf{S}$ - оператор электронного спина, гиромагнитное отношение $\gamma=g \mu_{\mathrm{B}}$ (где $g$ - электронный $g$-фактор,

$3^{*}$ Письма в ЖТФ, 2018, том 44, вып. 17 
$\mu_{\mathrm{B}}$ - магнетон Бора), $D$ - параметр, характеризующий расщепление тонкой структуры в аксиальном кристаллическом поле. Этот спиновый гамильтониан применим для обоих типов центров с $S=3 / 2$ и $S=1$, между которыми происходит кросс-релаксация, при этом $\gamma$ имеет одинаковую величину для этих центров.

Для ,яркого“ центра с $S=3 / 2$ уровни энергии $E$ в случае ориентации магнитного поля параллельно кристаллической оси $c$ (принятой нами за ось $z$ ) для проекций спина $M_{S}=+3 / 2,-3 / 2,+1 / 2$ и $-1 / 2$ имеют вид $E(+3 / 2)=(3 / 2) \gamma B+D, E(-3 / 2)=(-3 / 2) \gamma B+D$, $E(+1 / 2)=(1 / 2) \gamma B-D$ и $E(-1 / 2)=(-1 / 2) \gamma B-D$. Нас будут интересовать только переходы, которые приводят к изменению интенсивности ФЛ: $E(-3 / 2)-E(-1 / 2)=2 D-\gamma B, E(3 / 2)-E(1 / 2)=2 D+\gamma B$. Для „темного центра с $S=1$ уровни энергии для проекций $M_{S}=+1, \quad-1 \quad$ и 0 имеют вид $E(+1)=\gamma B+(1 / 3) D(T)$, $E(-1)=-\gamma B+(1 / 3) D(T), \quad E(0)=(-2 / 3) D(T)$. Разности энергий $E(+1)-E(0)=\gamma B+D(T), \quad E(0)-E(-1)=\gamma B-D(T)$. Выражение $D(T)$ подчеркивает зависимость параметра тонкой структуры от температуры.

Чтобы найти магнитные поля, соответствующие КР, необходимо приравнять разности энергий для центров с $S=3 / 2$ и с $S=1$, в результате получим выражение в виде абсолютной величины: $B=|D(T)-2 D| /(2 \gamma)$.

На рис. 1 показаны изменения интенсивности ФЛ $(\Delta \mathrm{PL})$ спиновых центров $V 2, V 3$ и $V 4$ в кристалле $15 R-\mathrm{SiC}$ (параметры $D$ спинового гамильтониана для ,ярких“ спиновых центров с $S=3 / 2$, обозначенных в литературе по их бесфононным линиям фотолюминесценции: $V 2$ $(886.5 \mathrm{~nm}), V 3(904 \mathrm{~nm})$ и $V 4(917 \mathrm{~nm})$ [5], соответственно равны 2.48, -0.2 и $0.9 \mathrm{mT})$ в зависимости от приложенного магнитного поля в области антипересечения спиновых уровней в основном состоянии (ground state LAC (GS LAC)), возбужденном состоянии (excited state LAC (ES LAC)) и в области кросс-релаксации (CR) между „яркими“ центрами $V 2, V 3$ и $V 4$ в квадруплетном спиновом состоянии и „темными“ центрами в триплетном спиновом состоянии. Штриховыми линиями представлены зависимости положения сигналов LAC и CR от температуры, которые могут быть использованы как градуировочные кривые, экспериментальные точки показаны в виде квадратов (ES LAC) и кружков (CR). Видно, что положение GS LAC не зависит от температуры. Для наглядности пунктиром показаны сигналы для GS LAC 


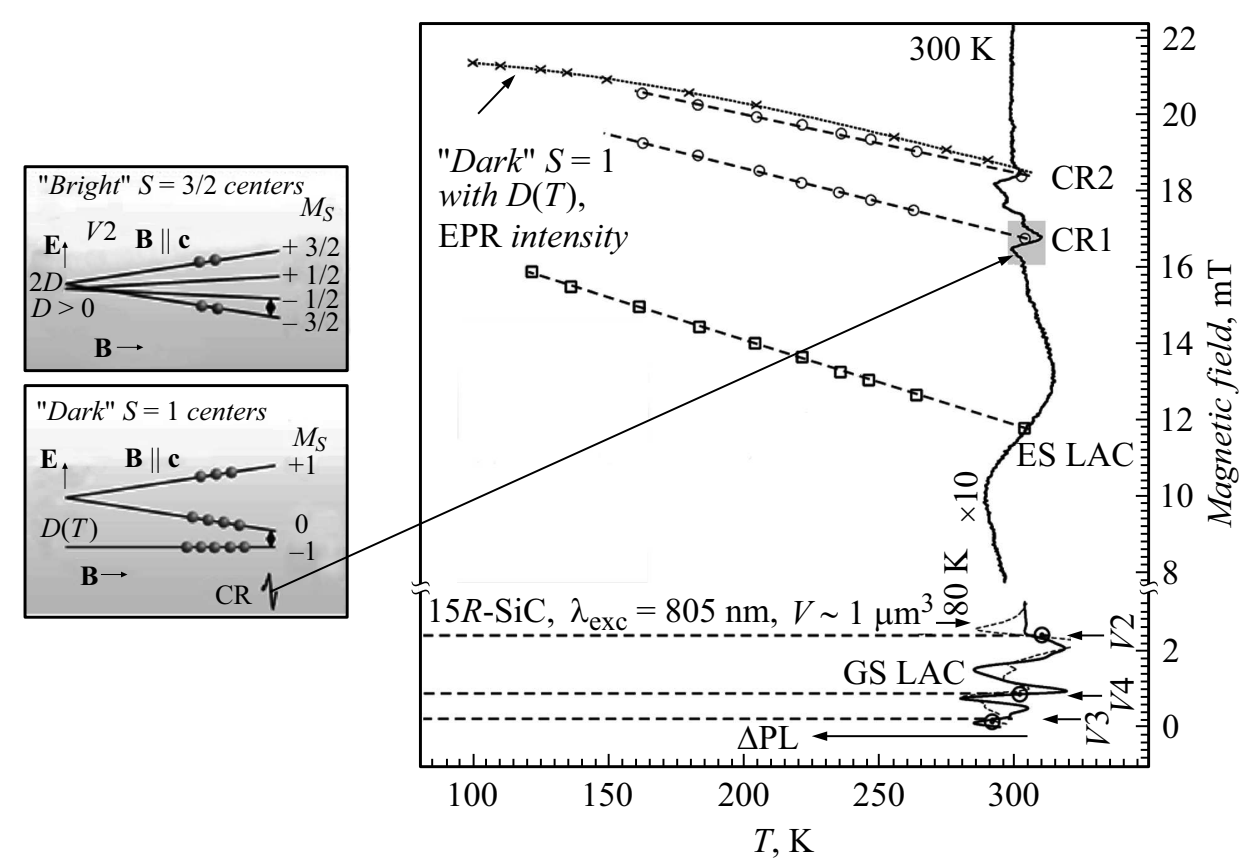

Рис. 1. Изменение интенсивности фотолюминесценции $(\Delta \mathrm{PL})$ спиновых центров в кристалле $15 R-\mathrm{SiC}$ в зависимости от приложенного магнитного поля (пояснения в тексте). 
при $80 \mathrm{~K}$. Для центра с $S=1$ параметр $D(T)$ сильно зависит от температуры (кривая с экспериментальными точками в виде крестиков на рис. 1). На вставках приведены спиновые подуровни для „яркого“ центра $V 2$ и для „темного“ центра с $S=1$. Стрелкой условно показано магнитное поле, при котором происходит кросс-релаксационный перенос спиновой поляризации, приводящий к изменению интенсивности ФЛ ,яркого“ центра $V 2$.

Согласно нашим измерениям в диапазоне $150-300 \mathrm{~K}$, зависимость положения KР $\left(B_{\mathrm{CR}}\right)$ от температуры в кристалле $15 R-\mathrm{SiC}$ (рис. 1) можно аппроксимировать линейной функцией $B_{\mathrm{CR}}=B_{0}-k T$, где $k-$ коэффициент линейной зависимости положения пика КР от температуры, $T$ - температура (в K). Для пиков CR1 и CR2, самых интенсивных и узких на рис. 1 , получаем $B_{\mathrm{CR} 1}=21.8 \mathrm{mT}-(0.017 \mathrm{mT} / \mathrm{K}) \cdot T$, $B_{\mathrm{CR} 2}=23.8 \mathrm{mT}-(0.018 \mathrm{mT} / \mathrm{K}) \cdot T$.

Для измерения температуры регистрируются зависимости изменения интенсивности ФЛ кристалла $\mathrm{SiC}$ от величины постоянного магнитного поля в области КР при разных температурах и строится градуировочная кривая для положения КР в магнитном поле в зависимости от температуры. Затем на поверхность кристалла $\mathrm{SiC}$ помещается исследуемый образец и измеряется магнитное поле в точке КР. С помощью известной градуировочной кривой определяется температура в области возбуждения ФЛ. Проводя сканирование поверхности образца, можно построить карту распределения температур по образцу.

Схема оптического квантового термометра представлена на рис. 2. Для создания и развертки магнитного поля используется источник постоянного тока (DC power supply), для формирования частотной модуляции - генератор низких частот (low frequency generator, LFG). Далее постоянный и переменный токи подаются на катушку электромагнита. Таким образом, созданное магнитное поле проникает в пластину кристалла $\mathrm{SiC}$, содержащую „яркие“ и „темные“ спиновые центры, и находится в тепловом контакте с исследуемым материалом. Спиновые центры реагируют на тепловой контакт с образцом, и мы можем регистрировать изменение ФЛ, „ярких“ спиновых центров с помощью объектива. Возбуждение осуществляется лазером с длиной волны $805 \mathrm{~nm}$. Сбор сигнала ФЛ происходит через полупрозрачное зеркало и светофильтр. Детектирование ФЛ осуществляется с помощью фотоприемника (photodetector) со спектральным диапазоном чувствительности 800-1100 nm. Далее сигнал с фотоприемника поступает на 

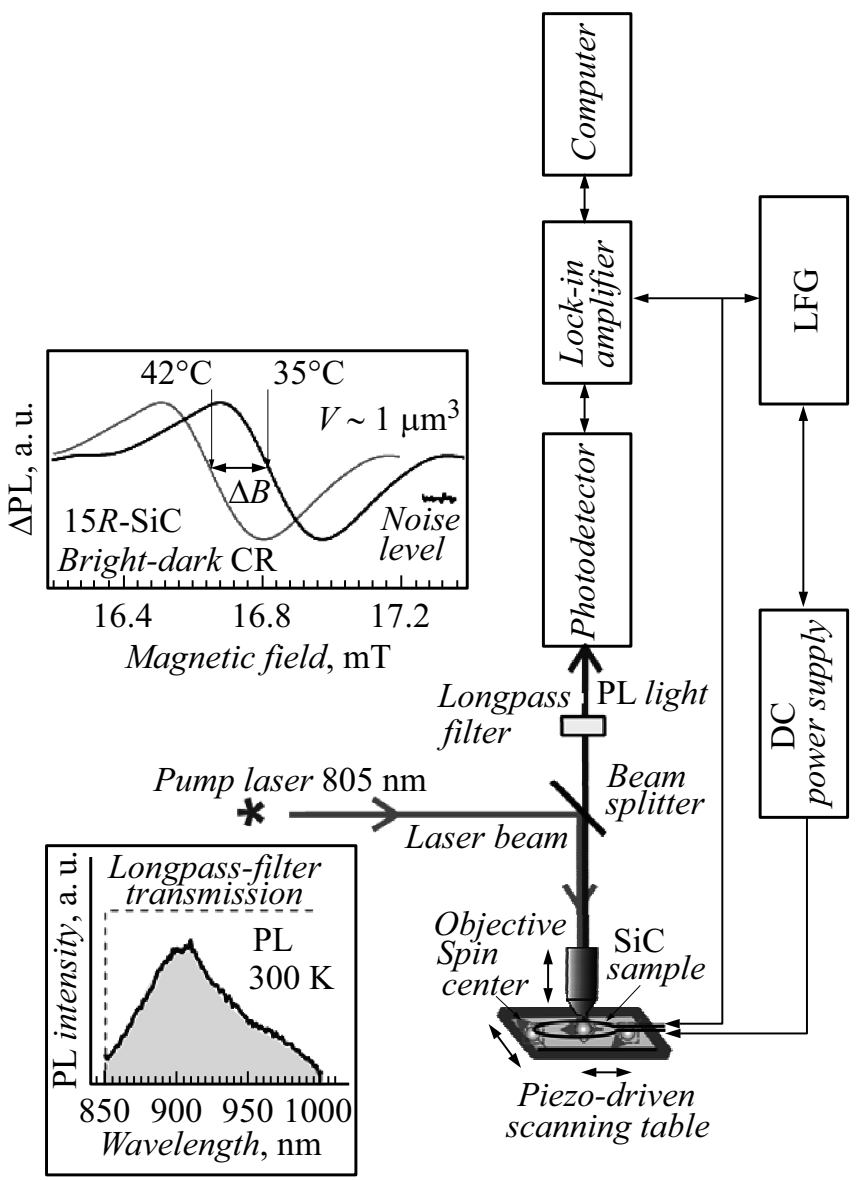

Рис. 2. Схема оптического квантового термометра (описание в тексте). На верхней вставке - расстояние $\Delta B$ между двумя положениями КР (сигнал CR1 выделен серым цветом на рис. 1) в магнитном поле для двух температур, характерных для биологических объектов. Показан шумовой уровень для одного скана в течение $0.1 \mathrm{~s}$. На нижней вставке - спектральная зависимость ФЛ при $300 \mathrm{~K}$, условно показано пропускание фильтра.

Письма в ЖТФ, 2018, том 44, вып. 17 
синхронный детектор (lock-in amplifier) и затем на блок управления и регистрации спектров (computer). Для получения пространственного разрешения используется сканирующий столик с пьезоэлементом, на который помещен активный материал в виде пластины кристалла $\mathrm{SiC}$ с взаимодействующими „яркими“ и „темными“ спиновыми центрами, показанными схематически. ФЛ фокусируется объективом микроскопа, который может сканировать в вертикальном направлении. На верхней вставке показан сдвиг сигнала CR1, который выделен серым цветом на рис. 1 , в магнитном поле при изменении температуры на $7^{\circ} \mathrm{C}$, характерном для биологических объектов (например, градуировка термометра для измерения температуры человека); $\Delta B-$ расстояние между двумя измерениями. Показан шумовой уровень для одного скана в течение $0.1 \mathrm{~s}$. Нижняя вставка представляет спектральную зависимость ФЛ при $300 \mathrm{~K}$, условно показано пропускание фильтра.

Величину $\Delta B_{\mathrm{CR}}$ можно затем преобразовать в температуру с помощью соотношения $\Delta T=-\Delta B_{\mathrm{CR}} / k$. Шумовой предел для одного сканирования с временем измерения $\sim 0.1 \mathrm{~s}$ составляет $3 \mu \mathrm{T}$ (исходя из наклона производной сигнала). Таким образом, можно оценить чувствительность, которая составляет приблизительно $100 \mathrm{mK} / \mathrm{Hz}^{1 / 2}$. При более низких температурах чувствительность примерно в 5 раз выше, так как интенсивность сигнала увеличилась примерно в 5 раз. При выборе более чувствительных детекторов и оптимизации оптической схемы можно достичь величины менее $10 \mathrm{mK} / \mathrm{Hz}^{1 / 2}$ в объеме менее $1 \mu \mathrm{m}^{3}$.

Работа выполнена при поддержке Министерства образования и науки (соглашение № 14.604.21.0200, идентификатор RFMEFI60417X0200).

\section{Список литературы}

[1] Neumann P., Jakobi I., Dolde F., Burk C., Reuter R., Waldherr G., Honert J., Wolf T., Brunner A., Shim J.M., Suter D., Sumiya H., Isoya J., Wrachtrup J. // Nano Lett. 2013. V. 13. P. 2738-2742.

[2] Kraus H., Soltamov V.A., Riedel D., Väth S., Fuchs F., Sperlich A., Baranov P.G., Dyakonov V., Astakhov G.V. // Nature Phys. 2014. V. 10. P. 157-162.

[3] Баранов П.Г., Бундакова А.П., Боровых И.В., Орлинский С.Б., Зондерван Р., Шмидт Я. // Письма в ЖЭТФ. 2007. Т. 86. В. 3. С. 231-235.

[4] Baranov P.G., Bundakova A.P., Soltamova A.A., Orlinskii S.B., Borovykh I.V., Zondervan R., Verberk R., Schmidt J. // Phys. Rev. B. 2011. V. 83. P. 125203.

Письма в ЖТФ, 2018, том 44, вып. 17 
[5] Soltamov A.V., Yavkin B.V., Tolmachev D.O., Babunts R.A., Badalyan A.G., Davydov V.Yu., Mokhov E.N., Proskuryakov I.I., Orlinskii S.B., Baranov P.G. // Phys. Rev. Lett. 2015. V. 115. P. 247602.

[6] Simin D., Soltamov V.A., Poshakinskiy A.V., Anisimov A.N., Babunts R.A., Tolmachev D.O., Mokhov E.N., Trupke M., Tarasenko S.A., Sperlich A., Baranov P.G., Dyakonov V., Astakhov G.V. // Phys. Rev. X. 2016. V. 6. P. 031014.

[7] Anisimov A.N., Simin D., Soltamov V.A., Lebedev S.P., Baranov P.G., Astakhov G.V., Dyakonov V. // Nature Sci. Rep. 2016. V. 6. P. 33301.

[8] Анисимов А.Н., Бабуни Р.А., Музафарова М.В., Бундакова А.П., Ильин И.В., Солтамов В.А., Романов Н.Г., Баранов П.Г. // Письма в ЖТФ. 2017. Т. 43. B. 7. C. 70-77.

[9] Baranov P.G., von Bardeleben H.-J., Jelezko F., Wrachtrup J. Magnetic resonance of semiconductors and their nanostructures: basic and advanced applications. Springer Ser. in Materials Science. V. 253. Springer-Verlag, 2017. $535 \mathrm{p}$.

[10] Павлов Н.М., Иглищын М.И., Косаганова М.Г., Соломатин В.Н. // ФТП. T. 9. B. 7. C. $1279-1285$.

Письма в ЖТФ, 2018, том 44, вып. 17 